\title{
Mobile Augmented Reality in Museums : Towards Enhancing Visitor's Learning Experience
}

\author{
Nehla Ghouaiel ${ }^{1}$, Samir Garbaya ${ }^{2}$, Jean-Marc Cieutat ${ }^{3}$ and Jean-Pierre Jessel $^{4}$
}

${ }^{1}$ IRISA laboratory, France

2 ENSAM Arts et Metiers ParisTech, France

${ }^{3}$ ESTIA Research laboratory, France

${ }^{4}$ IRIT laboratory, France

Abstract - This article presents the design and implementation of a handheld Augmented Reality (AR) system called Mobile Augmented Reality Touring System (M.A.R.T.S). The results of experiments conducted during museum visits using this system are also described. These experiments aim at studying how such a tool can transform the visitor's learning experience by comparing it to two widely used museum systems. First, we present the museum's learning experience and a related model which emerged from the state of the art. This model consists of two types of activity experienced by the observer of a work of art: sensitive and analytical. Then, we detail M.A.R.T.S architecture and implementation. Our empirical study highlights the fact that AR can direct visitors' attention by emphasizing and superimposing. Its magnifying and sensitive effects are well perceived and appreciated by visitors. The obtained results reveal that M.A.R.T.S contributes to a worthwhile learning experience.

Index Terms - Augmented reality; Mobile computing; Learning experience.

\section{INTRODUCTION}

Museums of Art and History are society's great archives of historical artifacts and artistic masterpieces. Museography aims to showcase the collections within an exhibition to visitors. These collections do not only contain concrete sources of information, but they also embody the knowledge of creators of artifacts and museum curators. In this context, it is considered that AR is an interesting tool that could be used to help visitors understand and appreciate the contents of an exhibition. AR also appears to be a suitable solution to reconcile the digital and real environments, since it visually superimposes information directly onto the exhibit as the user looks at the screen.

Over past decades, research has attempted to introduce AR to museums (Mase, Kadobayashi, and Nakatsu 1996) (Wagner, Schmalstieg, and Billinghurst 2006) (Miyashita et al. 2008). However, these studies have focused on evaluating AR devices or examining the potential benefits of introducing AR into the field of culture (Wagner, Schmalstieg, and Billinghurst 2006). They also investigate the necessary

E-mail: nehla.ghouaieleirisa.fr challenges in terms of technical constraints and audience (Miyashita et al. 2008).

Recently, few studies have attempted to examine the impact of AR on the overall visitor experience (Bationo Tillon et al. 2010) (Bationo Tillon, Marchal, and Houlier 2011). Since research works in museology (Pekarik, Doering, and Karns 1999) (Packer and Ballantyne 2002) (Kotler 1999) have distinguished several types of visitor experiences, this article focuses on a particular visitor experience : the learning experience. Indeed, learning experience is considered by the authors of these articles to be one of the most soughtafter experiences in museums. To our knowledge, there is no published research on the investigation of the impact of AR on learning experience in museums despite the fact that some AR implementations in museums started to appear in the recent past years. Therefore, the main question we intend to answer through this research is: how can AR transform the visitor's learning experience in an art and history museum? The findings of this study will be used in guiding the design of the user-approved version of M.A.R.T.S system to be setup on the Bayonne's Basque museum.

This article is organized as follows: first, a learning experience model which has emerged from the state of the art is described. Then, an overview of museum dedicated AR systems is presented in section 3. The proposals for supporting the visitor's learning experience and detailed description of M.A.R.T.S design and implementation is given in sections 4 and 5 . The method and the design of experiments are described in section 6 . The results are presented in section 7 and the section 8 is dedicated to the discussion and the review of the proposals. Finally, the conclusion is given in section 9 .

\section{LEARNING EXPERIENCE}

The visitor experience also called museum experience by Falk et al. (Falk and Dierking 1992), has for several years been both a relevant and complex research subject. Given that one of the main reasons for visiting museums is to discover and acquire new knowledge (Packer and Ballantyne 2002), hence this article focuses on the vistor's learning experience. In this context, it should be noted that learning in museums is voluntary, free and guided by the visitor's own choices. Several authors (Hooper-Greenhill et al. 2004) (Gammon 2003) have proposed models which enable the 


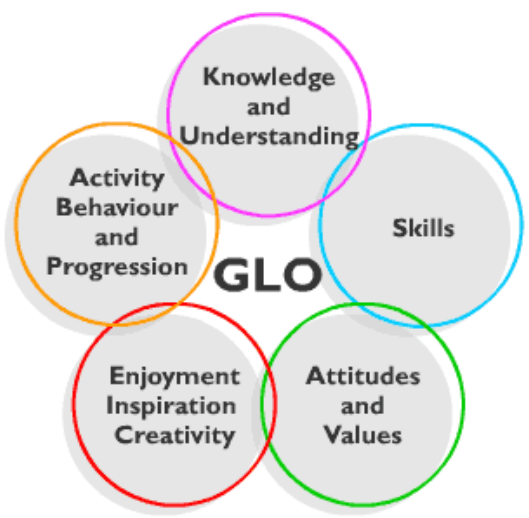

Figure 1: GLO (Generic Learning Outcomes) Model.

visitor's learning experience to be measured. This work is particularly interested in the GLO (Generic Learning Outcomes) model devised by Hooper-Greenhill et al. (HooperGreenhill et al. 2004). In fact, this model, in accordance with the work presented in (Bationo Tillon, Marchal, and Houlier 2011), highlights two dimensions: analytic and sensitive. These dimensions form the visitor experience which result from contact with exhibits. GLO was implemented by the UK Ministry of Museums, Libraries and Archives (MLA). Today it is widely used by several UK museums (Graham 2013) and was successfully applied by the mediation services of MLA. It is worth noting that the GLO model (figure 11 recognizes learning as an experience whose outcomes include: increased knowledge and understanding, additional skills, a change in values and attitudes, feelings of amusement, inspiration and creativity, and an incitement to activity and progression.

\section{State of The ART}

Today many museums around the world ${ }^{a}$ are aware of the potential advantages of using augmented reality. However, emerging technologies are generally introduced into the museum environment in several stages depending on progressive improvements made by research in the field. Many projects, prototypes and demonstrators have appeared over a period of almost two decades.

NaviCam (Rekimoto and Nagao 1995) is considered one of the pioneering systems of mobile augmented reality. NaviCam is based on colored barcodes to identify the object under concern. One of the uses of this prototype is to display textual information about an artwork in the form of virtual labels (digital messages) superimposed onto the camera view.

Virtuoso (Wagner, Schmalstieg, and Billinghurst 2006) is an educational game designed to be played by several museum visitors. The aim of the game is to sort a collection of artworks according to the date they were created. When players become confused, they can call on a virtual character having the same name as the application. In this paper,

\footnotetext{
${ }^{\mathrm{a}}$ http://www.museum-id.com/ideas.asp
}

the authors study the suitability of a handheld AR game to learn about the history of art by comparing it with more traditional variants (PC and paper). They present the results of a user study that demonstrates not only the effectiveness of AR for untrained users in collaborative edutainment, but also its fun-factor and suitability in museums. In general, users felt that the AR PDA system provided the most fun of the three conditions and would most improve the museum experience. However, a research study is still needed to explore the educational value of mobile AR applications and provide details about the design of these interfaces.

CEDRIC $b$ in collaboration with Renne's Museum of Fine Arts ${ }^{\mathrm{C}}$ developed an augmented reality guide prototype to assist visits (Damala et al. 2008). The prototype created is called AR Guide. Once the work of art is detected, AR Guide superimposes a menu with five items onto the work of art: description, technique, iconography, context and artist. For each item, the user can access different multimedia content enabling the work of art in question to be interpreted. The authors state that AR guide users criticized the redundancy of information presented via different media (eg. audio information was reproduced in text form).

The LDML project (Miyashita et al. 2008) started within the framework of collaboration between Paris'Louvre Museum and the Japanese company DNP (Dai Nippon Printing Co. Ltd ${ }^{d}$ This project provided two systems: a guidance system and a system for appreciating works of art. The first system enables users to be guided around the exhibition area (between the presentation halls) in order to follow a well determined route. The second system is used inside each presentation hall: it provides users with information helping them to assimilate the work of art's features. The presentation hall system namely provides the following functionalities:

- Display virtual text to interpret the work of art.

- Display the work of art's 3D model with the aim of enabling users to manipulate it. In this case, where AR is used, the authors mentioned that the back of exhibits can also be seen.

The experiments conducted with this system showed that visitors enjoyed using both functionalities. Nevertheless, larger group of subjects is necessary in order to see how AR technology used in a guidance system can help visitors finding their way around museum spaces, and how it facilitates or deepens their understanding of works of art.

GAMME (Bationo Tillon et al. 2010) (Bationo Tillon, Marchal, and Houlier 2011) is an acronym for "Guide Augmenté Mobile pour les Musées et Expositions" (Mobile Augmented Guide for Museums and Exhibitions). It is an industrial research project funded by the French Research Agency (ANR) that involves, amongst others, IRISA and Orange Labs ${ }^{f}$ In order to provide educational functions in-

\footnotetext{
${ }^{\mathrm{b}}$ Conservatoire National des Arts et Mtiers (National Conservatory for Arts and Skills), Paris, France

${ }^{c}$ http://www.mbar.org/index.php

${ }^{\mathrm{d}}$ http://www.dnp.co.jp/eng/

${ }^{\mathrm{e}} \mathrm{http} / / /$ www.irisa.fr/

${ }^{\mathrm{f}} \mathrm{http} / / / \mathrm{www}$.orange.com/fr/innovation
} 
side the museum, the prototype of the guide presented was based on the principles described by Goodman (Goodman 1987). The first GAMME prototype (Bationo Tillon et al. 2010), thanks to AR, has the following analytic functionalities:

- Find complementary colors in works of art.

- Highlight pentimento on a painting.

- Explore where the artists signature can be found on the back of paintings.

- Superimpose related works of art with the aim of comparing them.

The pentimento and comparison functionalities were advantageous for users. However, the other two issues were of no interest. Furthermore, users declared that they did not receive enough interpretations and information about the work of art. During 2011, the protagonists of GAMME, proposed a second prototype (Bationo Tillon, Marchal, and Houlier 2011). This prototype, completes the first by creating an emotional effect thanks to music, compositions and audio sequences. Nevertheless, it is worth noting that they kept the same analytic part as the first prototype.

Keil et al.(Keil et al. 2013) tried to create a cohesive narrative context in Athens's Acropolis Museum using the CHESS project (Keil et al. 2013)(Roussou et al. 2013) (Vayanou et al. 2012). This project aimed to provide four ways to digitally look at exhibits: virtual reconstruction of the original aspect; placement in the original location; visual highlighting of interesting details and annotations; and recreation of mythological appearances. It explores the use of personalized interactive storytelling experiences relating to museum exhibits delivered through mobile devices. In this work there are two types of mediation: the first is explorative and experience-driven through interaction and mixed media; the other is formal, more descriptive and explanatory. In (Keil et al. 2013) the authors attempted to evaluate the effect of AR incorporation in a storytelling context in museums. With their test scenarios they were able to prove the technological foundation of their concepts. However, they did not study the added value of AR storytelling for museum visits and did not evaluate its impact on visitor experience through an empirical study.

\section{M.A.R.T.S SYSTEM}

\section{IV.1. Our proposals}

Mediation techniques are a powerful way to shape vistor experience. Since mediation systems must be user-centered, how can we therfore design a museum guide system that takes into account visitors' preferences?

According to Bruner (Bruner 1960), representing knowledge requires several modes called "communication channels" by Hooper-Greenhill (Hooper Greenhill 1994). These ways of representing knowledge enable information to be passed on to others. Bruner (Bruner 1960) distinguishes three modes: symbolic, iconic and enactive. HooperGreenhill in the chapter "Museums: ideal learning environments" of his book (Hooper Greenhill 1994), insists on the importance of having these three modes for representing knowledge in museums : "The more opportunities for different modes of contact with ideas that are offered in any exhibition or presentation, the more possibilities will open up for the communication process"((Hooper Greenhill 1994), page 146).

Therfore, based on the concept of augmented reality, we propose to use these three ways of representing knowledge in our museum guide system called M.A.R.T.S. The enactive mode consists of learning through using real things, such as objects, or learning through people and events or through activities as explained in (Hooper Greenhill 1994). So, among other ways, the enactive mode can be achieved through a human guide lecturer. Taking into account that several research works on virtual and mixed environments have shown the co-presence ${ }^{g}$ imapact of virtual humans (Gerhard, Moore, and Hobbs 2005) (Luo, Shimada, and Sato 2014), we propose to simulate the presence of a human guide by a virtual human in M.A.R.T.S. As it will be detailed further, the enactive mode is represented by a virtual human guide simulating the presence of a human guide and supported by our "Selection" paradigm. The symbolic mode is conveyed by an interaction paradigm called "Documentation" and our "Reconstruction" paradigm refers to the iconic mode. The main purpose of these interaction plans is to help connecting digital information to exhibits in real time.

\section{IV.2. Virtual human guide}

Virtual humans represent an intuitive and natural means of communication reminiscent of communication between human beings. Indeed, based on a multimodal communication, a virtual guide can add speech and gestures (Bolt 1980).

The procedure leading for displaying the virtual human guide in the camera view is shown in the figure 2. A 3D conversational agent library for mobile platforms was developed in the framework of Aquitaine-Euskadi project. It is designed to be easily integrated with any mobile augmented reality engine despite its internal structure. The 3D graphics engine (avatar engine) of this library was built using Ogre3D (ref). For the animation, we adopted the engine developed by our project's partner Vicomtech h. The animation engine is the library's module to monitor the virtual human's movements and gestures. The structure of entire engine is described in (del Puy Carretero et al. 2012).

\section{IV.3. Visual interaction paradigm: Documentation}

The symbolic mode is very present in museums through using text. In Bayonne's Basque Museum ${ }^{\text {i }}$, for example, we can find labels with the name of the object, author and date of creation along with a description. It is therefore interesting to highlight textual information considered important by mediators (information contained in the label's title). From

\footnotetext{
${ }^{\mathrm{g}}$ Co-presence is having a feeling that one is in the same place as the other participants, and that one is collaborating with real people (Casanueva and Blake 2001).

in Vicomtech is an applied research centre: www.vicomtech.org

${ }^{\mathrm{i}}$ http://www.musee-basque.com/
} 


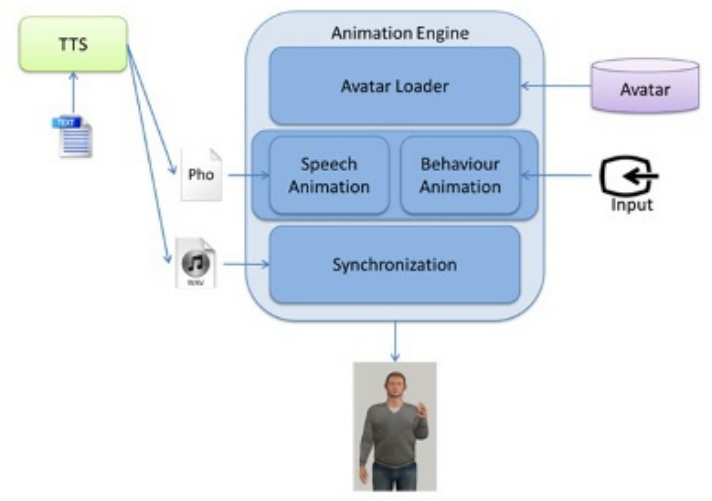

Figure 2: Structure of avatar engine.

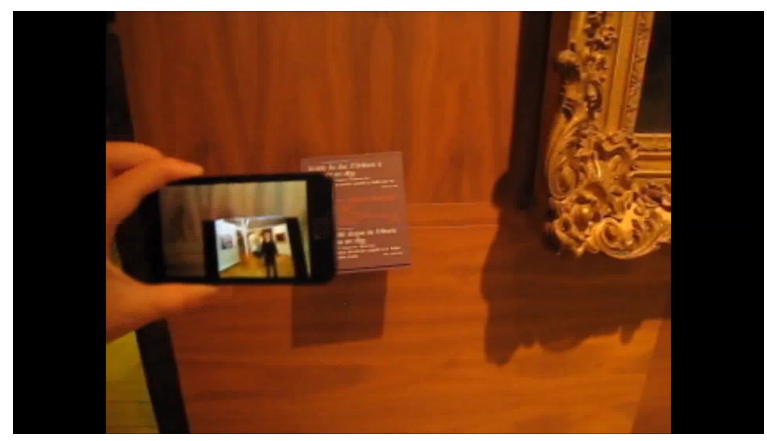

Figure 3: Virtual human guide in M.A.R.T.S system.

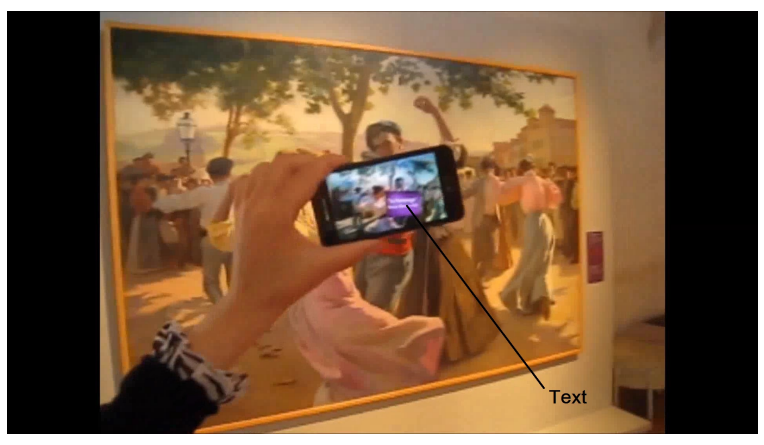

Figure 4: Virtual Documentation in M.A.R.T.S system

this perspective, augmented reality enables displaying a virtual text with the work's name and author (figure 4), in parallel with the virtual human guide's speech. This use of AR is essential, particularly, when the access to textual information is limited or not possible because of the crowd of visitors in front of a work of art. The display of virtual text is made using the OpenGL library.

\section{IV.4. Visual interaction paradigm: selection}

To explain an exhibit to visitors, we often need to refer to a specific part or area of it. For example, when interpreting the work entitled "Duke of Orlan's entry in 1839" (figure 5), in Bayonne's Basque museum, it is necessary to designate, amongst other things, the temporary triumphal arch made of wood. This is also the case for the work entitled "The confluence of the Nive and Adour rivers". For this specific painting, visitors must be able to locate "the monumental port of France" and the "Bust of Louis XV" in the painting's foreground. In the background, they can distinguish, amongst other things, "the Augustine bell" and the "Bulbous dome". It is obvious that when there is no visual reference, visitors are lost in exploring such items. They may also feel confused, even bored, and will lose the desire to continue looking at the work of art and following the interpretations.

To avoid this type of problems, we consider it is essential to implement a tool enabling users to easily distinguish the referenced elements on the exhibit. In this perspective, instead of implementing redundant explanations to locate them, we use an interaction paradigm that we called Selection. This simple interaction is representative of the deictic gesture, whose use, according to Spencer et al. (Kelly, Manning, and Rodak 2008) and Roth (Roth 2001), could be of great interest to consolidate the individual's understanding of the subject in question. Thus, the virtual human guide can simply point to a particular area (area of interest) on an exhibit. Visitors will therefore see an iconographic model (rectangle) that appears in front of their eyes in the area where they are supposed to look, thus avoiding any confusion or ambiguity (figure 5).

In order to implement this visual augmentation, we used the 2D image model of the area of interest. Feature points were extracted using SURF (Speeded Up Robust Features) method (Bay et al. 2008). Compared to SIFT (Juan and 


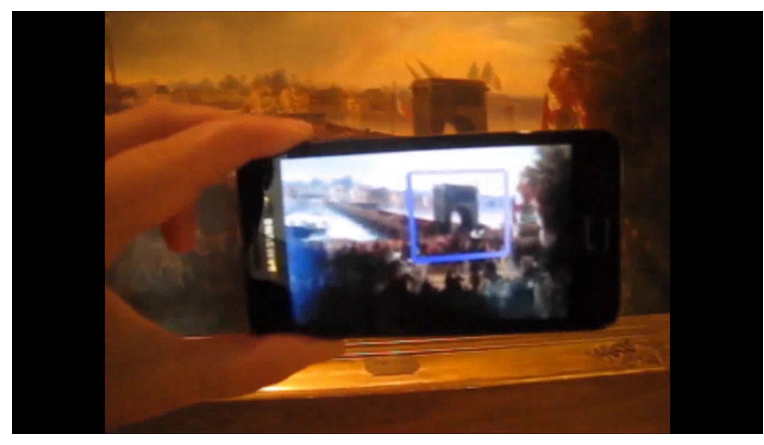

Figure 5: Visual Selection in M.A.R.T.S system.

Gwon 2009), SURF was basically chosen for its speed. To detect areas of interest on an exhibit (figuring in camera video stream), we propose the following method inspired by the work of Augereau et al. (Augereau, Journet, and Domenger 2013) :

- First, SURF keypoints are detected and relative descriptors (feature vectors) are extracted for both camera frame and $2 \mathrm{D}$ images in the database. Then, for each image model in the database, the similarity between the camera frame descriptor vectors $q$ and each descriptor $p$ from the model is computed. Each match $m\left(q_{i}, p_{j}\right)$ is considered as correct or incorrect based on the Euclidean distance $|\cdot, \cdot|_{2} \cdot$

$$
m\left(q_{i}, p_{j}\right)=\underset{j}{\arg \min }\left|q_{i}, p_{j}\right|_{2}
$$

In order to select the best match among candidate ones, we adopt the common approach relying on k-NN (nearest neighbor) classifier. Its complexity is however quadratic as a function of the number of keypoints. The multiple randomized kd-trees algorithm (Silpa-Anan and Hartley 2008) has the advantage of speeding up k-NN search. Thus, we used FLANN (Muja and Lowe 2009) library that provides an implementation of this algorithm where multiple kd-trees are searched in parallel. We note that for the randomized k-d trees, the split dimension is chosen randomly from the top 5 dimensions with the highest variance.

- Then, in order to determine the affine transformation between the model and the current frame, the homography (Agarwal, Jawahar, and Narayanan 2005) is computed. Homography uses the previously calculated point correspondences. At this stage, RANSAC algorithm (Fischler and Bolles 1981) is also used to eliminate outliers.

- The best model is selected according to the number of inliers. We are then able to proceed to the detection and selection(framing) of the area of interest on the exhibit.

- The four corners of the area of interest are calculated by applying the affine transformation (homography) computed in the previous step to the model ends. Those ends were represented by the following image coordinates ${ }^{j}$ : $(0,0) ;(0$, height); (width, height); (width, 0$)$.

\footnotetext{
${ }^{\mathrm{j}}$ Height and Width denote the height and width of the model.
}

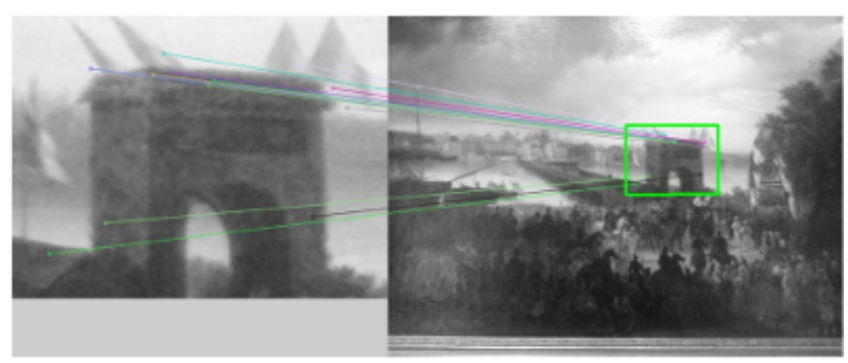

Figure 6: Implementation of Selection.

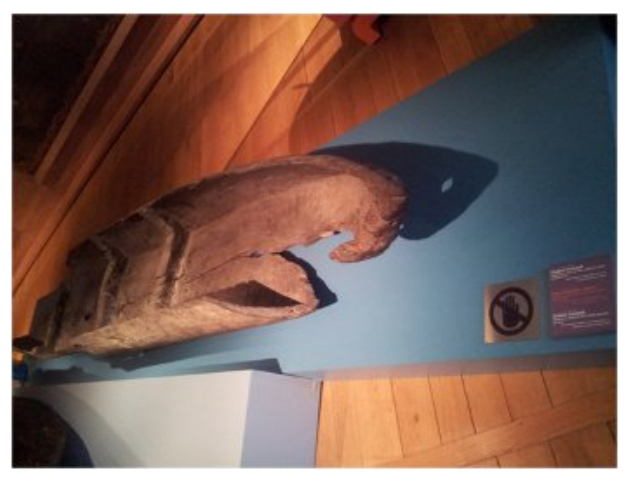

Figure 7: Deteriorated object (Dugout Barge) of middle age.

- Finally, OpenGL library functionalities are used to draw the quadrilateral connecting the four corners delimiting the area of interest.

\section{IV.5. Visual interaction paradigm: Reconstruction}

Thanks to augmented reality, a deteriorated object can be reconstructed in 3D (figure 7). In fact, virtual elements can be added in order to recreate the missing parts of an exhibit. Therfore, the aim is to show visitors what the original exhibit would have looked like. For this interaction, visitors will see the 3D representation of the object completely aligned with the real world. Clearly based on the principle of AR's enriched visibility, "Reconstruction" requires adequate 3D models to be owned upstream and registred on-line.

For this purpose, the exhibit's CAD model is superimposed on its remaining part. The implementation of the digital reconstruction requires robust real-time tracking of $3 \mathrm{D}$ objects with prior knowledge of the model. Hence, we are dealing with the problem of model based tracking with a monocular camera. The markerless tracking algorithm developed in this work was inspired by the work of Platonov et al. (Platonov et al. 2006), although it differs in several aspects that will be described later. The tracking algorithm is separated into two stages: offline (learning) stage and online stage.

In the first stage, key frames are created. To acquire a key frame, the marker must be placed in a known position in the world coordinate system. The camera pose provided 
by the marker is used to establish 2D-3D correspondences. Finally, the video frame (without the marker), 2D-3D correspondences and the camera pose provided by the marker are stored in a key frame structure.

The online stage is performed when the application has started, it comprises two phases: the initialization phase and the tracking phase. The first phase is responsible for matching the video frame to a key frame. Therefore, in the tracking phase we obtain the initial camera's pose $P_{t-1}$ at time $t-1$ (deduced from the key frame), video images $\left(I_{t-1}\right.$ and $I_{t}$ taken at times $t-1$ and $t$ respectively), as well as a 3D model $\mathrm{M}$ of the exhibit. The next step is to estimate the current camera pose $P_{t}$ as follows:

1. The set $V_{t-1}$ of 2D-3D correspondences is established (Platonov et al. 2006). First, 2D features are extracted from the frame image $I_{t-1}$. The CAD model M is projected (rendered) according to $P_{t-1}$. Then, extracted 2D features are projected onto the CAD model to establish 2D-3D correspondences.

2. The system performs frame-to-frame $2 \mathrm{D}$ feature tracking based on the KLT tracker (Lucas and Kanade 1981) which is a sparse optical flow algorithm. This is done by matching features of successive images $(t-1$ and $t)$. Instead of using Shi-Tomasi features (Sh and Tomasi 1994) as in (Platonov et al. 2006), we use SURF features to achieve stronger scale invariance. In fact, Shi-Tomasi features are reported (Platonov et al. 2006) to cause unbound error accumulation problems.

3. The set of 2D-3D correspondences $V_{t-1}$ is transformed into a new set $V_{t}$, which corresponds to the Image $I_{t}$.

4. The pose $P_{t}$ is computed using the POSIT algorithm (DeMenthon and Davis 1995).

Afterwards, we rely on OpenGL capabilities to project the CAD model onto the exhibit according to $P_{t}$. In order to solve the alignment issue, after each pose estimation step, all $3 \mathrm{D}$ points were projected onto the image plane. If the number of tracked features fell below a threshold ( $\approx 20$ points), the whole procedure was repeated by using the last estimated pose.

\section{Architecture OF M.A.R.T.S}

Figure 8 shows the hardware architecture of M.A.R.T.S As presented by the figure 9 , the system contains two different parts; one is installed on the user's smartphone and the other is deployed in a distant server.

The user application consists of three main modules. The AR module is responsible for displaying the computed augmentations. It relays on Virtual human and Detection/Tracking modules which related technical details are explained above. At the server side, the recognition module performs the identification of the exhibit captured by the smartphone camera. The database module consists of an SQL database containing 2D images, 3D models of exhibits and the corresponding descriptions. The communication between the user application and the server is ensured by IP network. For security reasons, the user authentification is

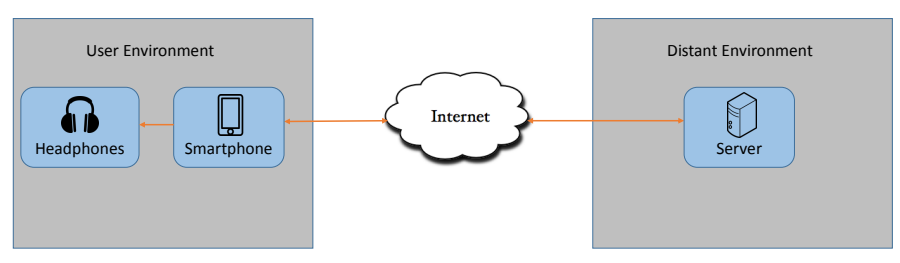

Figure 8: The hardware architecture of M.A.R.T.S

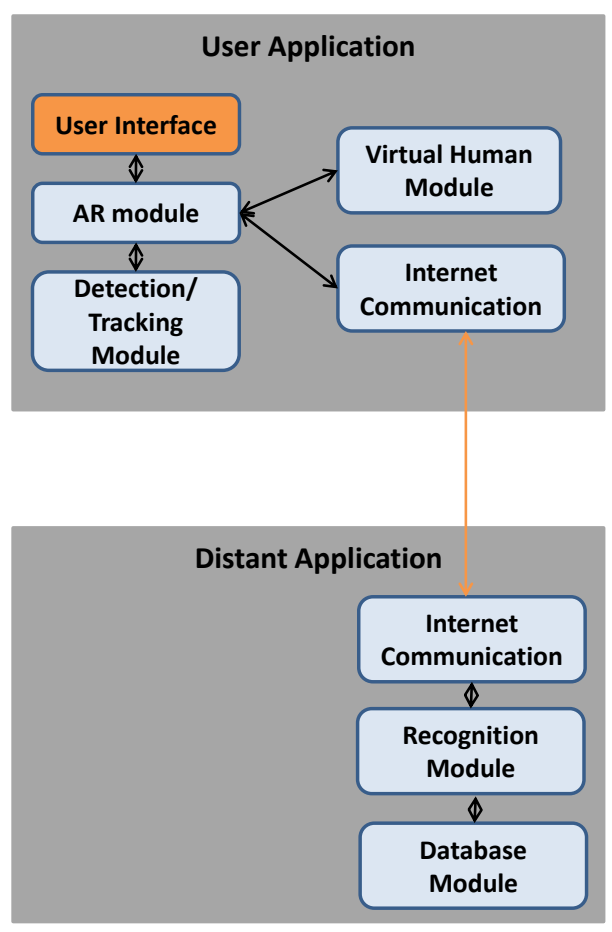

Figure 9: The software architecture of M.A.R.T.S 
performed by the OAuth ${ }^{\mathrm{k}}$ protocol and the data transfer is carried out by using SSL/TCL 1 protocol. In order to implement the user interface, Java and Android SDK are used. The others modules of the system are developped with $\mathrm{C}++$ language.

\section{EXPERIMENTAL DESIGN}

The experimental study described below was conducted in Bayonne's Museum of Art and History. The study concerned the use of M.A.R.T.S in real museum conditions. As mentioned previously, M.A.R.T.S user interface includes "Selection" and "Documentation" paradigms. The system also simulates the presence of a human guide represented by a virtual human. However, the "Reconstruction" paradigm is still under development and was not experimdented in the work described in this paper.

\section{VI.1. Method}

Objectives of the evaluation In the context of a guided museum visit, we intend to compare different means of communication used by visitors: labels $m$, audio-guide and the M.A.R.T.S system. It is worth mentioning that the first two systems are widely used in museums. Therfore, the experimental study intends to investigate whether AR has an advantageous impact on the learning experience and how it influences both sensitive and analytical aspects.

The study aims also at answering the following question: "Do these three different means of communication enable visitors to acquire the same level of knowledge?". In fact, quantifying the acquired knowledge after a museum visit is a fundamental aspect for a dedicated learning application. This aspect was highlighted in the work of Falk et al. (Falk and Storksdieck 2005). At the end of the tests, users were asked questions about their feelings and their appreciation in order to rank the different systems.

\section{VI.2. Procedure}

Participants 17 subjects ${ }^{n}$ took part in the study: 9 male and 8 female with an average age of 40 years. They are not involved in the research work described in this article. Furthermore, they were all visiting the museum for the first time and were very interested in Basque culture and the history of the city of Bayonne.

Experimental procedure In this study, we adopted the experimental procedure based on repeated-measures design

\footnotetext{
${ }^{\mathrm{k}}$ The OAuth protocol enables websites or applications (consumers) to access protected resources from a web service (service provider) via an API.

${ }^{1}$ Transport Layer Security (TLS) and its predecessor, Secure Sockets Layer (SSL), both frequently referred to as "SSL", are cryptographic protocols that provide communications security over a computer network.

${ }^{\mathrm{m}}$ Etiquette, written texts describing an object exhibited in a museum.

${ }^{\mathrm{n}}$ The used sample is expected to provide significant results because it presents the same characteristics as the studied population.
}

counterbalanced by conditions in order to determine the effect of each medium of mediation on the visitors knowledge acquisition. The problems relating to order effects and differences in experimental materials can be reduced by counterbalancing (Field 2005). In order to avoid the pitfalls of standard repeated measures designs we exposed the subjects to the different experimental conditions randomly. Moreover, the benefit of using such a design versus an independent design is to exclude the effects of individual differences that might occur if different people are requested to deal with the three conditions separately (Howitt and Cramer 2010). For this purpose, each subject used the three means of mediation : labels, audio-guide and M.A.R.T.S separately in a random order. For each of the three means of mediation, two of six exhibits, chosen randomly, were presented to the users, these two exhibits will have to be excluded from the list of the remaining exhibits to be explored by the others means of mediation and so on. This procedure ensures that each exhibit is explored only once during the experimentation. Because of the wide use of devices such as headphones and smartphones no training was necessary. The only instruction given to the subjects was to specify what is the means of mediation to be used for each exhibit. No time limit was imposed to the subjects and the experimentation was carried out using the three different medium of mediation without resting time between sessions. Finally, for ranking process, subjects were asked to fill in a questionnaire providing feedbacks on a set of a predetermined criteria.

Data Collection In this study two types of data are recorded: quantitative and qualitative. Quantitative data corresponds to the number of correct answers of questions about the exhibits. A question was asked for each exhibit (two for each means of mediation). Qualitative data is collected with the aim of estimating the learning experience. We used questions relating to the GLO model as a basis (Hooper-Greenhill et al. 2004) (Fuchs 2007). For each means of mediation being tested, the questions named from Q1 to Q6 were asked. To complete the questionnaire, the most used questions in AR literature (Dünser, Grasset, and Billinghurst 2008a) were asked. They are named from Q7 to Q9.

\section{RESULTS AND INTERPRETATION}

\section{VII.1. Number of correct answers}

The affects of three factors (labels, audio-guide, M.A.R.T.S) were studied for the quantitative dependent variable (the number of correct answers for the exhibits). For this, participants experienced all the experimental conditions representing the independant variable (means of mediation) in accordance with the previously described research plan. This condition is a repeated measurement design. In order to study the relationship between the means of mediation and the number of correct answers, the analysis of variance test (ANOVA) was used with a level of risk alpha of 0.05. The qualitative independent variable $X$ represents the means of 
ANOVA

\begin{tabular}{|l|c|c|c|c|c|}
\hline & $\begin{array}{c}\text { Sum of } \\
\text { squares }\end{array}$ & df & Mean Square & F & Sig. \\
\hline Between Groups &, 275 & 2 & 1,37 &, 463 &, 632 \\
Within Groups & 14,235 & 48 & 2,297 & & \\
Total & 14,510 & 50 & & & \\
\hline
\end{tabular}

Table 1: Inter-subject effect on numbers of correct answers.

communication (with 3 value levels) and the qualitative independent variable $\mathrm{Y}$ represents the number of correct answers. It should be noted that $\mathrm{Y}$ varies from 0 to 2 , since for each exhibit a single question was asked and each means of mediation is tested with the two exhibits. The statistical hypothesis $H_{0}$ (null hypothesis) is stated as the following: "The number of correct answers is equal for all means of mediation (rLabel =rAudio-guide = rM.A.R.T.S)". However, the alternative hypothesis $H_{1}$ corresponding to our research objectives is stated as : "the number of correct answers is different for each means of mediation". Table 1 presents the summary of the descriptive statistics of user tests.

The data analysis shows that participants, using the labels mediation mode, obtained a mean value of correct answers of $1.35(\sigma=0.606)$, a mean of $1.41(\sigma=0.507)$ for the audio-guide and $1.53(\sigma=0.514)$ for M.A.R.T.S. Therefore a gain in favor of M.A.R.T.S in terms of the mean of correct answers, of approximately $6 \%$ compared to the audio-guide and $9 \%$ compared to the labels.

The ANOVA result yielded that $\mathrm{F}(2,48)=0.463$ and $\mathrm{p}=$ 0.632 . The obtained $\mathrm{F}$ value was 0.463 which is less than the critical value of 3.49 given by the Fisher-Snedecor table. Thus, the null hypothesis $\mathrm{H} 0$ is true. The obtained $\mathrm{P}$ value $(0.632)$ is higher than the 5\% threshold. Therefore, There is no statistically significant difference between the correct answsers obtained by the three means of mediation, although according to the analysis of means there is a gain in the favor of M.A.R.T.S.

\section{VII.2. Subjective evaluation}

Question $1(\mathrm{Q} 1)$ : "How did your perception of the art and history of the Basque Country change?" $(0=$ Very unfavorable to $14=$ Highly favorable $)^{\circ}$ The table 2 shows the following scores for the three experimented mediation tools: M.A.R.T.S $=9.82$, Audio-Guide $=8.24$ and Label $=6.47$. M.A.R.T.S has obtained the highest mean score, which highlights that this system can improve the vistors perception of Basque art and history.

Question 2 (Q2) concerns the feeling of having learnt something new. The table 2 shows the following scores for the three experimented tools: M.A.R.T.S $=11.47$, AudioGuide $=9.24$ and Label $=8.12$.These results highlight a slightly higher value for the Audio-guide compared to the Labels. It is worth noting, that all visitors considered M.A.R.T.S very adequate for learning in museums.

${ }^{\circ}$ This evaluation scale is mentioned in the work carried out by (Dünser, Grasset, and Billinghurst 2008b)
Question 3 (Q3) deals with how much the subject was surprised when using any of the three mediation systems $(0=$ Very disappointing $14=$ Very exceptional $)$. The table 2 shows the following scores for the three experimented tools: M.A.R.T.S $=11$, Audio-Guide $=8.67$ and Label $=6.29$. Here too M.A.R.T.S obtained the best rank. Visitors considered that M.A.R.T.S makes the exhibition more gripping. This is certainly related to augmented reality since subjects were not familiar with this technology and were often surprised by the integration of virtual objects in the real scene.

Question $4(\mathrm{Q} 4)$ is about whether the subjects will continue the exploration of the art and history of the Basque Country and the city of Bayonne after their visit $(0=$ Not at all to $14=$ Most certainly). The table 2 shows the following scores for the three experimented tools: M.A.R.T.S $=8.29$, Audio-Guide $=7.65$ and Label $=7.41$. The averages relating to the three systems show that they all induce the same motivation of the visitors to carry out further research about the museum's themes.

Question 5 (Q5):“Do you feel you are able to recognize certain symbols and motifs from the art of the Basque Country?" ( $0=$ Not at all to $14=$ Entirely). The table 2 shows the following scores for the three experimented mediation tools: M.A.R.T.S $=8.59$, Audio-Guide $=8.35$ and Label $=7.41$. The results demonstrate that visitors think they have acquired the same skills with all the three systems being experimented.

Question 6 (Q6): "Is it easy to make the link between the indications for the means of mediation and the work of art?" ( $0=$ Very difficult to $14=$ Very easy). The table 2 shows the following scores for the three experimented tools: M.A.R.T.S $=11.65$, Audio-Guide $=8.35$ and Label $=5.76$. Results show the superiority of M.A.R.T.S and the Audioguide. In fact, with these two systems, visitors can situate themselves in front of the work of art and therefore were able to more easily make the link between it and the audio comments. On the one hand, the difference between M.A.R.T.S and the Audio-guide mainly relates to the efficiency of the designation by the "Selection" interaction paradigm. This result perfectly illustrates the usefulness of image-based means of communication.

Question 7 (Q7) concerns the ease of use $(0=$ Very difficult to $14=$ Very easy). The table 2 shows the following scores for the three experimented tools: M.A.R.T.S $=10.82$, AudioGuide $=9.94$ and Label $=7.88$. The ranking highlights the inferiority of the Labels compared to the Audio-guide and M.A.R.T.S. This emphasises that subjects prefer to listen to descriptions relating to works of art rather than reading them in a text.

Question 8 (Q8) deals with the degree of comfort experienced with each system $(0=$ Very uncomfortable to $14=$ Very comfortable). The table 2 shows the following scores for the three experimented mediation tools: M.A.R.T.S $=9.35$, Audio-Guide $=10.59$ and Label $=6.47$. Audio-Guide obtained the best rank because it is a hands-free system.

Finally, in the case of question 9 (Q9): "How much stress did you experience for each means of mediation?" $(0=$ Very low to $14=$ Very high). The table 2 shows the following scores for the three experimented mediation tools: M.A.R.T.S $=3.82$, Audio-Guide $=3.88$ and Label $=3.47$. 


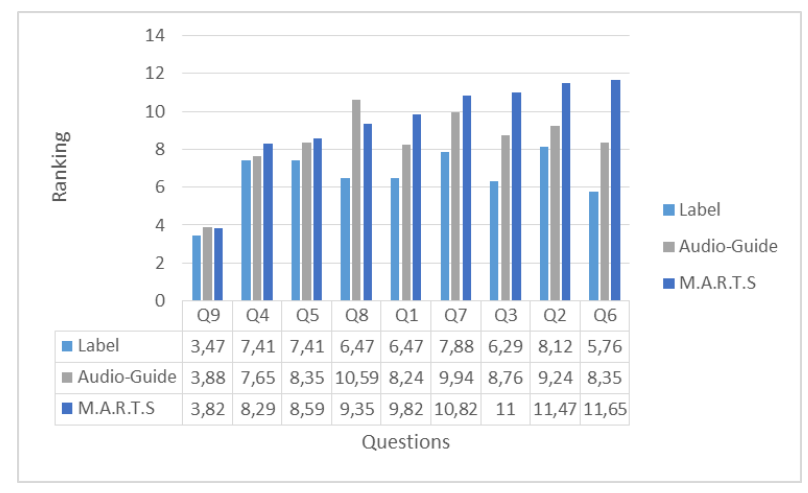

Table 2: Means of the scores attributed by subjects.

With regard to the stress criteria, it was noticed that there was no significant difference between the experimented medium, additionally none of them was considered stressful.

\section{DISCUSSION}

The initial results showed that there is no statistically significant difference in terms of the number of correct answers about exhibits, despite the apparent gain in favor of M.A.R.T.S, obtained by the analysis of means, $(9 \%$ compared to Labels and 6\% compared to the Audio-guide). Based on the observations of users' behaviors during the experiments, it is noticed that some subjects have made extra effort in handling the system's interface. This leads to the conclusion that user's attention was influenced by the nature of the interface rather than following the interpretations. Hence, further studies are needed in order to improve the ergonomic design of M.A.R.T.S.

However, it was noticed that subjects were predisposed to use the Audio-guide and M.A.R.T.S which is confirmed by the subjects' answers about how easily they acquired information (question 7). Indeed, in the case of both systems, the subjects highly appreciated being able to listen to the descriptions about the work of art instead of having to read them (in the case of Labels). Nevertheless, there is still a difference between Audio-guide and M.A.R.T.S with regard to the ranking especially in question 6 . In fact, subjects declared that it was easier with M.A.R.T.S to identify the parts of a work of art referenced by audio comments. This is due to the "Selection" interaction paradigm, enabling areas of interest about the work of art to be referenced and establish a link between the comments and the reality. It is also worth of mentioning that using AR creates a feeling of surprise (question 3 ) to the majority of the participants. Consequently, they found the exhibition more gripping.

Furthermore, despite the light weight of smartphone, subjects expressed the wish to keep it in their pocket. They prefer to take it out only for pointing at the exhibit when it is needed to display the "Selection" and "Documentation" paradigms. Therefore, in order to improve the ease of use, simulating the co-presence of a human guide can be reduced to the conversational aspects (audio mode only). This obser- vation complies with the answers to the question 8 in which subjects considered that the Audio-guide is more comfortable to use than the M.A.R.T.S system.

Finally, the outcome of this research work allows concluding that M.A.R.T.S is a suitable tool for a descriptive, object-oriented approach because it helps the visitor to connect digital information to physical objects. The study proved that the proposed interaction paradigms enable visitors to pay more attention to the exhibit details. Hence, they showed more interest in works of art. Additionally, this study provides experimental information feedbacks to direct further research aiming at introducing augmented reality systems in museums as the case of Labels and Audioguide.

\section{CONCLUSION}

This paper describes a Mobile Augmented Reality Touring System (M.A.R.T.S), as a tool for assisting vistors of museums. M.A.R.T.S complies with the principles of learning in a museum, proposed by Falk et al. (Falk and Dierking 2000) (Falk and Storksdieck 2005) and Hooper-Greenhill et al. (Hooper-Greenhill et al. 2004) (Hooper Greenhill 1994).

This research study explored the relevance of M.A.R.T.S as an interface enabling the description of museum exhibits. The evaluation study showed that using "Selection" and "Documentation" paradigms facilitates the acquisition of knowledge. Moreover, it was easier for visitors to establish the binding between the descriptions and the exhibits. This leads to the conclusion that AR can direct the visitor's attention by emphasizing and superimposing. Its magnifying and sensitive effects are well perceived and appreciated by visitors, from this perspective, M.A.R.T.S contributes to a valuable learning experience.

However, visitors have pointed out some drawbacks concerning the ergonomic aspects of M.A.R.T.S system interface. The visualization of the virtual guide could be the origin of the deviation of users attention. In order to ease the use of the interface, the simulation of the human guide copresence will be limited to the conversational mode (audio only). Additionally, further studies will also be conducted to improve the ergonomics of the user interface design. The module of the reconstruction paradigm will be integrated in M.A.R.T.S and further experimentations will be necessary to validate the efficiency of this mediation system and its added value for the acquisition of knowledge for the visitors of museums.

\section{Acknowledgements}

M.A.R.T.S (Mobile Augmented Reality Touring System) was developed as part of the Aquitaine-Euskadi project 2012-2014. The authors acknowledge the contribution of the French and Spanish governments for funding this project.

\section{REFERENCES}

[Agarwal, Jawahar, and Narayanan 2005] Agarwal, A.; Jawahar, C. V.; and Narayanan, P. J. 2005. A survey of planar homography estimation techniques. (2005). 
[Augereau, Journet, and Domenger 2013] Augereau, O.; Journet, N.; and Domenger, J.-P. 2013. Semi-structured document image matching and recognition. Document Recognition and Retrieval (2013).

[Bationo Tillon et al. 2010] Bationo Tillon, A.; Marchand, E.; Laneurit, J.; Servant, F.; Marchal, I.; and Houlier, P. 2010. A day at the museum: An augmented fineart exhibit. IEEE International Symposium on Mixed and Augmented Reality 2010 Arts, Media, and Humanitites Proceedings 9:69-70.

[Bationo Tillon, Marchal, and Houlier 2011]

Bationo Tillon, A.; Marchal, I.; and Houlier, P. 2011. Mobile augmented reality in the museum: Can a lace-like technology take you closer to works of art? IEEE International Symposium On Mixed and Augmented Reality - Arts, Media, and Humanities (ISMAR-AMH). 41-47.

[Bay et al. 2008] Bay, H.; Ess, A.; Tuytelaars, T.; and Van Gool, L. 2008. Speeded-up robust features (surf). Computer Vision and Image Understanding (CVIU) 110:346-359.

[Bolt 1980] Bolt, R. A. 1980. Put-that-there : Voice and gesture at the graphics interface. SIGGRAPH Comput. Graph. 14:262-270.

[Bruner 1960] Bruner, J. S. 1960. The process of education. Harvard University Press (1960).

[Casanueva and Blake 2001] Casanueva, J. S., and Blake, E. H. 2001. The effects of avatars on co-presence in a collaborative virtual environment. Annual Conference of the South African Institute of Computer Scientists and Information Technologists (SAICSIT2001). Pretoria, South.

[Damala et al. 2008] Damala, A.; Cubaud, P.; Bationo, A.; Houlier, P.; and Marchal, I. 2008. Bridging the gap between the digital and the physical: design and evaluation of a mobile augmented reality guide for the museum visit. Proceedings of the 3rd International Conference on Digital Interactive Media in Entertainment and Arts 120-127.

[del Puy Carretero et al. 2012] del Puy Carretero, M.; Ardanza, A.; García, S.; Díez, H.; Oyarzun, D.; and Ruiz, N. 2012. Improving gestural communication in virtual characters. Proceedings of the 7th international conference on Articulated Motion and Deformable Objects 7:69-81.

[DeMenthon and Davis 1995] DeMenthon, D. F., and Davis, L. S. 1995. Model-based object pose in 25 lines of code. International Journal of Computer Vision 15:123-141.

[Dünser, Grasset, and Billinghurst 2008a] Dünser, A.; Grasset, R.; and Billinghurst, M. 2008a. A survey of evaluation techniques used in augmented reality studies. ACM SIGGRAPH ASIA 2008 courses 1-27.

[Dünser, Grasset, and Billinghurst 2008b] Dünser, A.; Grasset, R.; and Billinghurst, M. 2008b. A survey of evaluation techniques used in augmented reality studies. $1-27$.
[Falk and Dierking 1992] Falk, J., and Dierking, L. 1992. The museum experience. Whalesback Books (1992).

[Falk and Dierking 2000] Falk, J. H., and Dierking, L. D. 2000. Learning from museums: Visitor experiences and the making of meaning. AltaMira Press (2000).

[Falk and Storksdieck 2005] Falk, J., and Storksdieck, M. 2005. Using the contextual model of learning to understand visitor learning from a science center exhibition. Science Education 89:744-778.

[Field 2005] Field, A. 2005. Discovering Statistics Using SPSS. SAGE Publications.

[Fischler and Bolles 1981] Fischler, M. A., and Bolles, R. C. 1981. Random sample consensus: a paradigm for model fitting with applications to image analysis and automated cartography. Commun. ACM 24:381-395.

[Fuchs 2007] Fuchs, J. 2007. Generic learning outcomes as a strategic tool for evaluating learning impact. TICOMCECA Conference, Vienna (2007).

[Gammon 2003] Gammon, B. 2003. Assessing learning in museum environment a practical guide for museum evaluators. London : science museum report (2003).

[Gerhard, Moore, and Hobbs 2005] Gerhard, M.; Moore, D.; and Hobbs, D. 2005. Close encounters of the virtual kind: Agents simulating copresence. Applied Artificial Intelligence: An International Journal 19:393-412.

[Goodman 1987] Goodman, N. 1987. Of mind and other matters. Harvard University Press (1987).

[Graham 2013] Graham, J. 2013. Evidencing the impact of the glos 2008 2013. The University of Leicester's School of Museum Studies, report (2013).

[Hooper-Greenhill et al. 2004] Hooper-Greenhill, E.; Dodd, J.; Moussouri, T.; Jones, C.; Pickford, C.; Herman, C.; Morrison, M.; Vincent, J.; and Toon, R. 2004. Measuring learning outcomes in museums, archives and libraries: The learning impact research project (lirp). International Journal of Heritage Studies 10:151-174.

[Hooper Greenhill 1994] Hooper Greenhill, E. 1994. Museums and their visitors. Routledge. New York (1994).

[Howitt and Cramer 2010] Howitt, D., and Cramer, D. 2010. Introduction to Research Methods in Psychology. Prentice Hall; 3 edition.

[Juan and Gwon 2009] Juan, L., and Gwon, O. 2009. A comparison of sift, pca-sift and surf. International Journal of Image Processing (IJIP) 3:143 -152.

[Keil et al. 2013] Keil, J.; Pujol, L.; Roussou, M.; Engelke, T.; Schmitt, M.; Bockholt, U.; and Eleftheratou, S. 2013. A digital look at physical museum exhibits: Designing personalized stories with handheld augmented reality in museums. Digital Heritage International Congress (DigitalHeritage) 2:685 - 688 .

[Kelly, Manning, and Rodak 2008] Kelly, S. D.; Manning, S. M.; and Rodak, S. 2008. Gesture gives a hand to language and learning: Perspectives from cognitive neuroscience, developmental psychology and education. Language and Linguistics Compass 2:569-588. 
[Kotler 1999] Kotler, N. 1999. Delivering experience: marketing the museum's full range of assets. Museum News 30-39.

[Lucas and Kanade 1981] Lucas, B. D., and Kanade, T. 1981. An iterative image registration technique with an application to stereo vision. Proceedings of the 7th International Joint Conference on Artificial Intelligence 2:674-679.

[Luo, Shimada, and Sato 2014] Luo, H.; Shimada, H.; and Sato, K. 2014. Interactive co-presence environment with mixed reality using remote and local avatar. International Journal on Computer Science and Engineering 6.

[Mase, Kadobayashi, and Nakatsu 1996] Mase, K.; Kadobayashi, R.; and Nakatsu, R. 1996. Metamuseum: A supportive augmented-reality environment for knowledge sharing. in ATR Workshop on Social Agents: Humans and Machines 107-110.

[Miyashita et al. 2008] Miyashita, T.; Meier, P. G.; Tachikawa, T.; Orlic, S.; Eble, T.; Scholz, V.; Gapel, A.; Gerl, O.; Arnaudov, S.; and Lieberknecht, S. 2008. An augmented reality museum guide. IEEE International Symposium on Mixed and Augmented Reality (ISMAR) 103-106.

[Muja and Lowe 2009] Muja, M., and Lowe, D. G. 2009. Fast approximate nearest neighbors with automatic algorithm configuration. In VISAPP International Conference on Computer Vision Theory and Applications 331-340.

[Packer and Ballantyne 2002] Packer, J., and Ballantyne, R. 2002. Motivational Factors and the Visitor Experience: A Comparison of Three Sites. Curator: The Museum Journal 45:183-196.

[Pekarik, Doering, and Karns 1999] Pekarik, A. J.; Doering, Z. D.; and Karns, D. A. 1999. Exploring Satisfying Experiences in Museums. Curator: The Museum Journal 42:152-173.

[Platonov et al. 2006] Platonov, J.; Heibel, H.; Meier, P.; and Grollmann, B. 2006. A mobile markerless ar system for maintenance and repair. Proceedings of the 5th IEEE and ACM International Symposium on Mixed and Augmented Reality 105 -108.

[ref ] http://www.ogre3d.org.

[Rekimoto and Nagao 1995] Rekimoto, J., and Nagao, K. 1995. The world through the computer: Computer augmented interaction with real world environments. Proceedings of the 8th Annual ACM Symposium on User Interface and Software Technology 29-36.

[Roth 2001] Roth, W. M. 2001. Gestures: their role in teaching and learning. Review of Educational Research 365-392.

[Roussou et al. 2013] Roussou, M.; Katifor, A.; Pujol, L.; Vayanou, M.; and Egglestone, S. R. 2013. A life of their own: museum visitor personas penetrating the design lifecycle of a mobile experience. Conference on $\mathrm{Hu}$ man Factors in Computing Systems 547-552.
[Sh and Tomasi 1994] Sh, J., and Tomasi, C. 1994. Good features to track. 1994 IEEE Conference on Computer Vision and Pattern Recognition (CVPR'94) 593 - 600.

[Silpa-Anan and Hartley 2008] Silpa-Anan, C., and Hartley, R. 2008. Optimised kd-trees for fast image descriptor matching. volume $60,1-8$.

[Vayanou et al. 2012] Vayanou, M.; Karvounis, M.; Kyriakidi, M.; Katifori, A.; Manola, N.; Roussou, M.; and Ioannidis, Y. 2012. Towards personalized storytelling for museum visits. 6th International Workshop on Personalized Access, Profile Management, and Context Awareness in Databases (2012).

[Wagner, Schmalstieg, and Billinghurst 2006] Wagner, D.; Schmalstieg, D.; and Billinghurst, M. 2006. Handheld ar for collaborative edutainment. Proceedings of the 16th International Conference on Advances in Artificial Reality and Tele-Existence 85-96.

\section{Sample of questions}

- What is the difference between the form of the catholic cross and the basque cross?

- What the traditionnal basque clothing is composed of?

- What are the characteristics of the basque danse?

- What the temporary arch made for the Duke of Oralan's entry represented? 